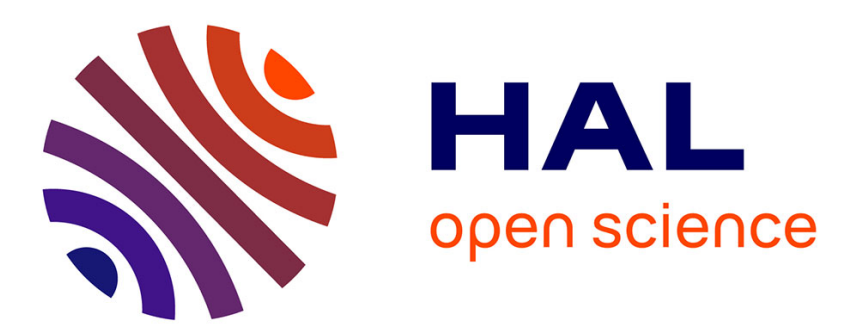

\title{
Inertial mass transport and capillary hydraulic jump in a liquid foam microchannel
}

Alexandre Cohen, Nathalie Fraysse, Jean Rajchenbach, Médéric Argentina, Yann Bouret, Christophe Raufaste

\section{To cite this version:}

Alexandre Cohen, Nathalie Fraysse, Jean Rajchenbach, Médéric Argentina, Yann Bouret, et al.. Inertial mass transport and capillary hydraulic jump in a liquid foam microchannel. Physical Review Letters, 2014, 112, pp.218303. 10.1103/PhysRevLett.112.218303 . hal-01010821

\section{HAL Id: hal-01010821 \\ https://hal.science/hal-01010821}

Submitted on 20 Jun 2014

HAL is a multi-disciplinary open access archive for the deposit and dissemination of scientific research documents, whether they are published or not. The documents may come from teaching and research institutions in France or abroad, or from public or private research centers.
L'archive ouverte pluridisciplinaire HAL, est destinée au dépôt et à la diffusion de documents scientifiques de niveau recherche, publiés ou non, émanant des établissements d'enseignement et de recherche français ou étrangers, des laboratoires publics ou privés. 


\title{
Inertial mass transport and capillary hydraulic jump in a liquid foam microchannel
}

\author{
Alexandre Cohen, ${ }^{1, *}$ Nathalie Fraysse, ${ }^{1}$ Jean Rajchenbach, ${ }^{1}$ \\ Médéric Argentina, ${ }^{2,3}$ Yann Bouret, ${ }^{4}$ and Christophe Raufaste ${ }^{4}$ \\ ${ }^{1}$ Université Nice Sophia Antipolis, CNRS, LPMC, UMR 7336, 06100 Nice, France \\ ${ }^{2}$ Université Nice Sophia Antipolis, CNRS, INLN, UMR 7335, 06560 Valbonne, France \\ ${ }^{3}$ Institut Universitaire de France, 75005 Paris, France \\ ${ }^{4}$ Université Nice Sophia Antipolis, CNRS, LPMC, UMR 7336, 06300 Nice, France
}

\begin{abstract}
We report a capillary-inertial flow in an unbounded liquid microchannel -a Plateau border in a liquid foam- and discuss its relevance for foam drainage. In contrast to the case of jets of cylindrical geometry, which are susceptible to the Rayleigh-Plateau instability, in this channel any liquid perturbation is dispersed instead of amplified. The geometry of the Plateau border provides an effective negative surface tension that triggers the liquid redistribution. The dynamics is perturbationindependent and leads to the formation of a hydraulic jump, at the micron-millimeter scale, traveling at constant velocity.
\end{abstract}

The rheological behavior of liquids in unbounded geometries (i.e. without rigid boundary conditions) is not well understood. Examples are jets, films, and liquid foams, the dynamics of which is driven either by gravity or geometrical inhomogeneities (capillary suction). The drainage of soap foams under gravity has in particular been investigated $[1-5]$. In such media, the liquid is assumed to flow through the network of channels called "Plateau borders" (PBs). The most controlled experiment, called forced drainage, consists in pouring liquid at constant flow rate on top of a foam column: a swollen region of constant liquid fraction is observed to develop from the top and travel downward at constant velocity [3]. Macroscopic measurements at the scale of the foam sample have been satisfactorily accounted for by effective-medium models of flow through porous media, assuming a low Reynolds number. The so-called drainage equation is used $[1,2,5]$. Capillary suction can be neglected within the swollen region, which displays a homogeneous liquid fraction $[1,2,5]$, however it can no longer be disregarded for determining the exact profile of the front zone $[3,6,7]$ where capillary effects become important, as evidenced by the few microgravity experiments [8]. Generally speaking, few experiments have been devoted to the study of the local flow at the scale of one elementary cell (i.e. one bubble), and all of them dealt with steady regimes $[1,9]$.

The present study investigates transient flows through a Plateau border with capillarity as the only driving force. This is achieved experimentally by injecting a droplet into a single Plateau border which is maintained horizontally to eliminate gravity effects.

We use a straight, $15 \mathrm{~mm}$-long Plateau border created by dipping a triangular-prism frame into a soapy solution. The frame is positioned so that the cross-section of the three films that hold the PB adopts the Y-shape shown in Fig. 1a. The setup geometry is analogous to the one used by Piroird and Lorenceau to study oil imbibition inside an aqueous foam [10]. The cross-section in Fig. 1b is typical of Plateau borders in foams. The radius of curvature of the PB is controlled by continuously injecting liquid at one upper-corner of the frame. Two kinds of surfactant solutions are probed in the experiments: Solution A was obtained from a commercial dishwashing liquid, Solutions B were obtained by dissolving TTAB (Tetradecyl trimethyl ammonium bromide) into de-ionized water and adding various amounts of glycerol in order to vary the viscosity. Solutions A and B are characterized by tangential stress-free interfaces (high surface mobility limit) $[9,11]$. We inject extra liquid into the PB by releasing from above a small spherical droplet of the same surfactant solution. After release, the droplet rebounds on the $\mathrm{PB}$, stabilizes and finally coalesces with the PB. In these experiments, the droplet radius ranges from $r=0.2 \mathrm{~mm}$ to $1.8 \mathrm{~mm}$, while the initial radius of curvature of the PB ranges from $R_{i}=0.1$ to $1.3 \mathrm{~mm}$. The relaxation of the PB-with-drop is visualized by means of a fast camera.

There are two different dynamical regimes. A viscous regime is observed for large bulk viscosities or small radii of curvature of the Plateau border: the bulge corresponding to the fluid in excess spreads with time and the liquid is slowly evacuated into the PB (Fig.1c). The PB thickness profile is smooth (Fig.1f) and presents a diffusivelike spreading dynamics (data not shown). In contrast, an inertial regime is observed for small solution viscosities and regular or large radii of curvature of the Plateau border. The liquid in excess does not spread smoothly, but is evacuated by dilating the initial $\mathrm{PB}$ in a front-like manner (Fig.1d,e). A sharp traveling jump (Fig.1g,h) separates the dilated part of the $\mathrm{PB}$, which has a uniform and constant radius of curvature $R_{j}$, from the notyet-perturbed part characterized by the initial radius of curvature $R_{i}$. This jump travels with a constant and substantial velocity $(\sim 0.1-1 \mathrm{~m} / \mathrm{s})$.

We now turn to the transition between the two dynamical regimes described above, as well as the reason for referring to them as "viscous" versus "capillary-inertial". Five parameters, namely $\rho, \eta, \gamma, r$ and $R_{i}$, are relevant in the system, which yields only two independent dimensionless numbers. The first one is the ratio of the droplet 


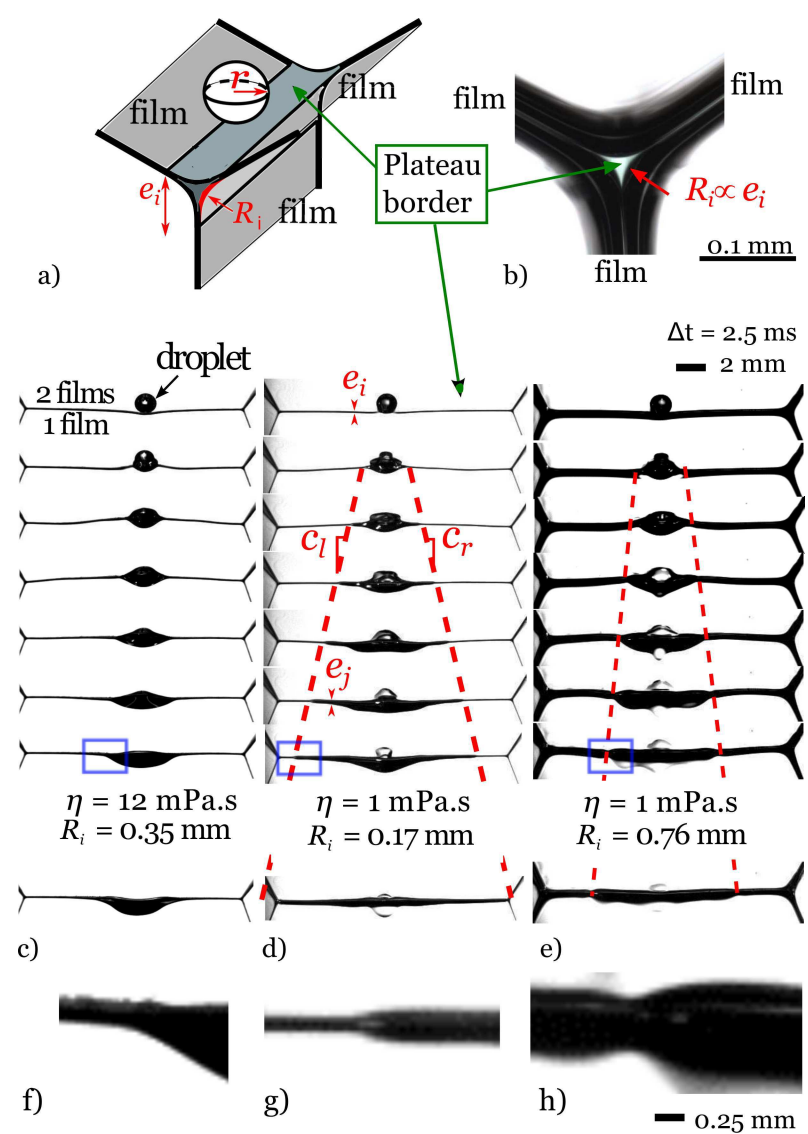

FIG. 1. a) Experimental setup: the Plateau border is held by three soap films. A drop is released from above and enters in contact with the Plateau border. b) Cross-section of the initial Plateau border observed through one of its extremity. At rest, the cross-section is well approximated by the tangential contact of three arcs of circles of same radius equal to $R_{i} . e_{i}$ is the apparent thickness of the PB and is proportional to its radius of curvature $R_{i}$. c-e) Three sets of snapshots of the longitudinal view of the $\mathrm{PB}$ that illustrate the different behaviors observed. $\Delta t=2.5 \mathrm{~ms}$ between consecutive images ( $5 \mathrm{~ms}$ between the last two images of each series). c) Viscous regime for a high viscosity TTAB solution. d) Inertial regime for the less viscous TTAB solution. $c_{l}\left(c_{r}\right)$ holds for the velocity of the front that travels toward the left (right). Red dashed lines are guides to the eyes to follow the front position. e) Same as d) but for a thicker initial PB. f-h) Enlargement of the edge of the perturbed part of the $\mathrm{PB}$. The enlarged zones are pinpointed by the blue boxes drawn on the penultimate images of the snapshot series in c-e).

size to the PB size, $r / R_{i}$. The second one is the socalled Ohnesorge number - often used to deal with freeinterface problems in fluid mechanics [12]. This number can be defined as the ratio of a capillary-inertial velocity $c_{0}=\sqrt{\gamma / \rho R_{i}}$ to a capillary-viscous velocity $\gamma / \eta$ :

$$
O h=\frac{\eta}{\sqrt{\rho \gamma R_{i}}} .
$$

The Ohnesorge number is actually the inverse of the Reynolds number $R e$ based on $c_{0}$. The experiments re-

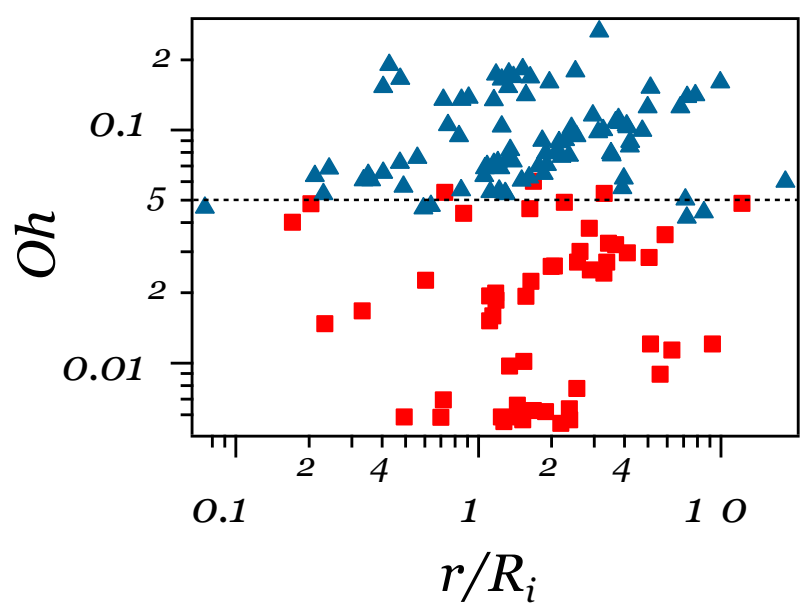

FIG. 2. Ohnesorge number as function of ratio of droplet size to PB size. Red squares ( $\square$ ) and blue triangles ( $\mathbf{\Delta})$ stand for the inertial regime and for the viscous regime, respectively. The straight horizontal line $(O h=0.05)$ highlights the transition between the two regimes.

ported in Fig. 2 show a well-defined transition between the two regimes, for a critical value $O h=0.05$, or equivalently $R e=20$, independently of $r / R_{i}$. For $O h$ larger than $0.05(R e<20)$, viscosity is observed to dominate, whereas for Oh smaller than $0.05(R e>20)$, a capillaryinertial regime is observed. We emphasize that this transition is droplet-size-independent.

This new capillary-inertial dynamics needs to be investigated because all current foam drainage models consider viscous flows only. In the rest of this paper, we concentrate on this regime and its relevance for foam drainage dynamics.

Experimental results obtained in the inertial regime are reported in Fig.3 for Solution A and for the less viscous Solution B. The front velocity (computed as the average over the right and left swelling fronts velocities, see Fig.1d) strongly decreases when the initial radius of curvature of the PB increases (Fig.3a); a power law interpolation gives exponents of $-0.51 \pm 0.05$ and $-0.50 \pm 0.02$ for the two solutions, respectively. The radius of curvature $R_{j}$ upstream of the jump increases linearly with the initial radius of curvature $R_{i}$; the best linear fits are $R_{j}=(1.9 \pm 0.1) R_{i}$ and $R_{j}=(1.5 \pm 0.1) R_{i}$ for the two solutions, respectively (Fig.3b). All the experiments in the inertial regime show similar behavior: the properties of the traveling jump are determined by the properties of the initial PB and do not depend on the amplitude of the perturbation. Within our experimental range, the initial radius of the droplet does not appear to play a significant role neither on the front velocity nor on the radius of curvature of the perturbed region of the $\mathrm{PB}$. This is true as long as the droplet acts as a liquid reservoir that can sustain the jump geometry.

We propose a model based on two assumptions. First, 

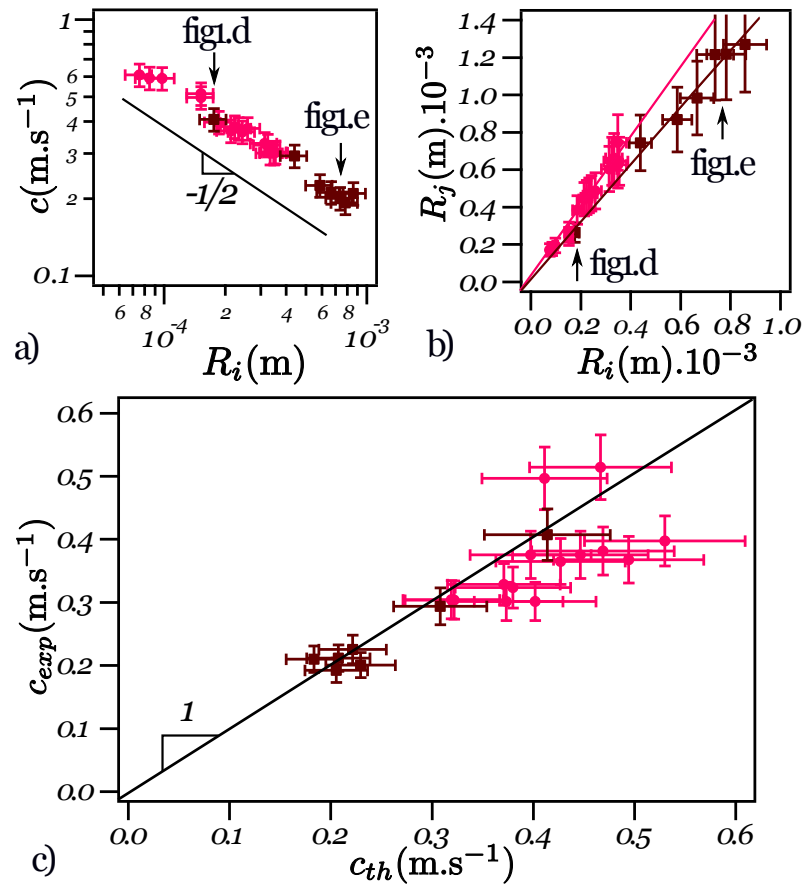

FIG. 3. Characterization of the inertial regime for Solution A (light red circles $\bullet$ ) and for the less viscous Solution B (dark red squares $\square$ ). a) Front velocity as a function of the initial radius of curvature $R_{i}$ of the Plateau border, for various radii of the coalescing droplets (logarithmic scales). The black straight line drawn on the graph has a slope -0.5. b) Radius of curvature of the dilated part of the Plateau border as a function of the initial radius of curvature $R_{i}$ of the Plateau border (same experiments as in a)). Solid lines are the best linear fits. c) Measured front velocity compared to the front velocity predicted by the model based on the capillary hydraulic jump geometry (Eq. 6). The data points that correspond to the experiments shown in Fig.1d,e are pinpointed by arrows on a) and b).

a)

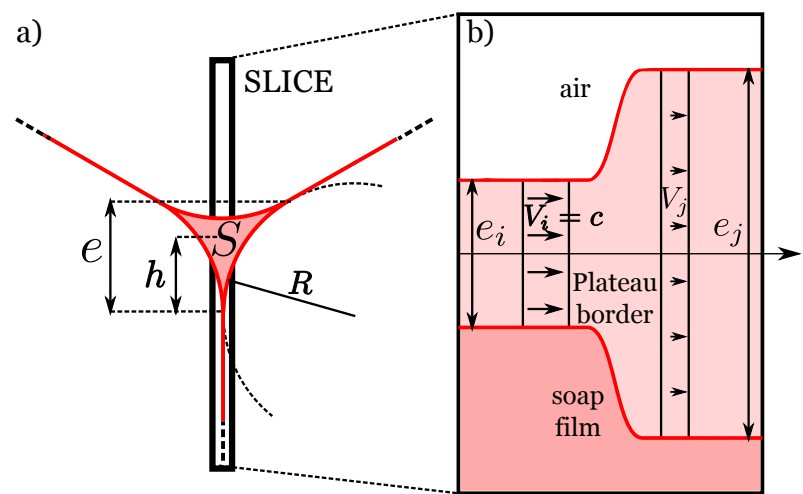

FIG. 4. a) Sketch of the cross-section of the PB held by the three liquid films. Geometrical considerations lead to $S=(\sqrt{3}-\pi / 2) R^{2}$ and $h=R / \sqrt{3}$. b) Representation of the capillary hydraulic jump geometry (based on experimental observations, Fig. 1g,h) in the reference frame of the moving front. the Reynolds number based on the front velocity ( $0.2 \mathrm{~m} / \mathrm{s})$ and on the PB size $(\sim 0.5 \mathrm{~mm})$ is of order 100 . Therefore we will consider the flow as inertia-dominated. Secondly, we assume a plug-flow inside the PB, at least far from the front zone, because of the high mobility of the surfactants at the interfaces [11]. The geometry of the jump zone is analogous to the one of a hydraulic jump triggered by gravity [13] if considered in the reference frame of the moving front $\mathcal{R}_{f}$.

A sketch of this geometry is displayed in Fig. 4, together with the notations used. Balance equations relate to $\mathcal{R}_{f}$. We assume that the liquid is initially at rest in the reference frame of the lab, $\mathcal{R}$, thus $V_{i}=c$, where $c$ is the velocity of the traveling front in $\mathcal{R}$. The mass balance equation applied on both sides of the jump gives:

$$
R_{i}^{2} c=R_{j}^{2} V_{j}
$$

The momentum difference rate accross the jump thus is:

$$
\Delta p / \Delta t=\rho S_{i} c^{2}-\rho S_{j} V_{j}^{2}=\rho S_{i} c^{2}\left(\left(R_{i} / R_{j}\right)^{2}-1\right),
$$

where $S_{k}=(\sqrt{3}-\pi / 2) R_{k}^{2}$ is the BP cross-section area (Fig. 4). This negative rate must be balanced by the net capillary force $F_{j}-F_{i}$ that applies on the capillary jump. Three contributions to the capillary force have to be taken into account (ordered as such in the following formula): (1) capillary pressure force on the PB crosssection, (2) line tension on the PB contour and (3) line tension on the three holding films (note that each film has two liquid-gas interfaces):

$$
\frac{F_{j}-F_{i}}{\gamma}=\left(\frac{S_{j}}{R_{j}}-\frac{S_{i}}{R_{i}}\right)+\pi\left(R_{j}-R_{i}\right)-6\left(h_{j}-h_{i}\right)
$$

which reduces to

$$
\frac{F_{j}-F_{i}}{\gamma}=-(\sqrt{3}-\pi / 2)\left(R_{j}-R_{i}\right)
$$

It is noteworthy that the film contribution is always negative and is dominant in the capillary net force. It is also remarkable that the expression of the driving force (Eq. 5) is equivalent to the one of a capillary pressure with a negative surface tension $-\gamma[14]$ : this reflects the fact that an increase in the $\mathrm{PB}$ radius is energetically favorable as it results in a decrease of the interfacial area of the holding films that prevails over the concomitant increase of the interfacial area of the PB. For that reason, the capillary pressure only (the same holds for the tension force) would never support a capillary hydraulic jump in classical 1D geometries, like jets.

Combining Eqs. (3) and (5) leads to the following intrinsic relation linking geometry and dynamics:

$$
c\left(R_{i} / R_{j}\right)=c_{0} \frac{1}{\sqrt{R_{i} / R_{j}\left(1+R_{i} / R_{j}\right)}},
$$


where $c_{0}=\sqrt{\gamma / \rho R_{i}}$ is the capillary-inertial velocity previously introduced. We use the value given by the experimental data (Fig. $3 \mathrm{~b}$ ) for the ratio $R_{i} / R_{j}$ in order to compute the velocity predicted by the model (Eq. 6). The comparison with the front velocity that was measured is shown in Fig. 3c. Despite some dispersion, the agreement is satisfactory since the model comprises no free parameter.

Consider now liquid foams. In the dry foam limit, the radius of curvature of the Plateau borders is related to an effective bubble diameter. For instance, the Kelvin structure leads to the approximate relation: $\Phi \simeq 1.32\left(R_{i} / D\right)^{2}$ [15], where $D$ is the bubble diameter and $\Phi$ is the liquid fraction of the foam. The Ohnesorge number thus is:

$$
O h=1.07 \frac{\eta}{\sqrt{\rho \gamma D \Phi^{1 / 2}}}
$$

This expression yields an estimate of the critical bubble radius $D_{c}$ above which the inertial regime may be observed. Common values for liquid foams $(\rho=1000$ $\mathrm{kg} . \mathrm{m}^{-3}, \gamma=30 \mathrm{mN} . \mathrm{m}^{-1}, \eta=1 \mathrm{mPa} . \mathrm{s}$ and $\left.\Phi=0.01\right)$ lead to $D_{c}=0.15 \mathrm{~mm}$. One has to be cautious when extrapolating data from experiments performed on model foams that have characteristic sizes significantly larger than the ones of usual foams. More importantly, a large set of experiments reported in the literature should exhibit a fast liquid flow along the Plateau borders. This is true for drainage studies $[1,8,16,17]$, but also for other liquid redistribution cases such as bubble rearrangements [18]. However, since this process is fast compared to other processes involved (dissipation in vertices for drainage studies, dissipation inside the opening film during bubbles rearrangements), the dynamics of the system is ruled by the latter and capillary suction is not the origin of the limiting time scale. Nevertheless, it should be taken into account in gravity-free environment or to describe the shape of the transition zone in drainage studies. Note that the analysis performed in this study is valid only for tangential stress-free interfaces. Experiments performed with surfactants of low surface mobility reveal other behaviors (data not shown). We have never observed the hydraulic jump geometry in this case.

In summary, we have designed a local scale experiment and shown the occurrence of a fast mass transport regime along Plateau borders in liquid foams. This regime is triggered by capillary suction and is characterized by a capillary-inertial velocity which scales as $\sqrt{\gamma / \rho R_{i}}$, where $\gamma, \rho$ and $R_{i}$ are the surface tension of the liquid, its den- sity and the radius of curvature of Plateau border, respectively. We have given a phenomenological criterion for this regime to occur in foams, in terms of interfacial properties of the liquid, foam liquid fraction and bubble radius. It will be crucial to look closely at the exact profile of the front zone during forced drainage and to scrutinize the Plateau borders during bubble rearrangements like $\mathrm{T} 1$ processes to detect the capillary-inertial regime.

We gratefully acknowledge fruitful discussions with members of the GDR 2983 Mousses et Emulsions (CNRS). Franck Celestini is thanked for insightful comments. This work was supported by a research funding granted by the University of Nice Sophia Antipolis.

* corresponding author: Alexandre.Cohen@unice.fr

[1] S. A. Koehler, S. Hilgenfeldt, and H. A. Stone, Langmuir 16, 6327 (2000).

[2] G. Verbist, and D. Weaire, Europhys. Lett. 26, 631 (1994).

[3] D. Weaire, N. Pittet, S. Hutzler, and D. Pardal, Phys. Rev. Lett. 71, 2670 (1993).

[4] A. Saint-Jalmes, Soft Matter 2, 836 (2006).

[5] S. Cohen-Addad, R. Höhler, and O. Pitois, Annu. Rev. Fluid Mech. 45, 241 (2013).

[6] P. Grassia, J.J. Cilliers, S.J. Neethling, and E. VenturaMedina, Eur. Phys. J. E 6, 325 (2001).

[7] P. Grassia, S.J. Neethling, and J.J. Cilliers, Eur. Phys. J. E 8, 517 (2002).

[8] H. Caps, S.J. Cox, H. Decauwer, D. Weaire, and N. Vandewalle, Colloids Surf. A 261, 131 (2005).

[9] O. Pitois, C. Fritz, and M. Vignes-Adler, Colloids Surf. A 261, 109 (2005).

[10] K. Piroird, and E. Lorenceau, Phys. Rev. Lett. 111, 234503 (2013).

[11] C. Raufaste, A. Foulon, and B. Dollet, Phys. Fluids 21, $053102(2009)$.

[12] J. Eggers, and E. Villermaux, Rep. Prog. Phys. 71, 036601 (2008).

[13] L. Rayleigh, Proc. R. Soc. Lond. A 5, 324 (1914).

[14] J.-C. Géminard, A. Zywocinski, F. Caillier, and P. Oswald, Phil. Mag. Lett. 84, 199 (2004).

[15] D. Weaire, and S. Hutzler, The Physics of Foams (Oxford University Press, Oxford, 1999).

[16] A. Saint-Jalmes, S. Marze, H. Ritacco, D. Langevin, S. Bail, J. Dubail, L. Guingot, G. Roux, P. Sung, and L. Tosini, Phys. Rev. Lett. 98, 058303 (2007).

[17] V. Carrier, S. Destouesse, and A. Colin, Phys. Rev. E 65, 061404 (2002)

[18] A.-L. Biance, A. Delbos, and O. Pitois, Phys. Rev. Lett. 106, 068301 (2011). 